\title{
Industrial Safety Applications Using Wireless Access Panels
}

\author{
Sakam Nagi Reddya, Dr. A A Ansari ${ }^{\text {b }}$ \\ ${ }^{a}$ Research Scholar, Dept. of Electronics and Communication Engineering, \\ Sri Satya Sai University of Technology \& Medical Sciences, Sehore, Bhopal Indore Road, Madhya Pradesh, India \\ ${ }^{\mathbf{b}}$ Research Guide, Dept. of Electronics and Communication Engineering, \\ Sri Satya Sai University of Technology \& Medical Sciences, Sehore, Bhopal Indore Road, Madhya Pradesh, India
}

Article History: Received: 11 January 2021; Accepted: 27 February 2021; Published online: 5 April 2021

\begin{abstract}
In large numbers of the industrial sectors, wireless networking technologies are acquiring rapid endorsement on account of their cost-adequacy, improved unwavering quality, and adaptability. A wireless network can be characterized as a network of devices, meant as hubs. Their essential usefulness is to corporately detect, accumulate, measure, and distribute data in the general climate. Wireless methods communication without the utilization of wires other than the recieving wire, the Ethernet, and the ground replacing wires. For a few industrial activities, wireless technology bears the cost of savvy and compelling availability arrangements. Utilizing wireless technology there are numerous advantages, for example, distant offices, cycles and field activity which brings about improving productivity, personal time, quicker and more accurate data assortment.
\end{abstract}

\section{Introduction}

WSNs are steadily embraced in the industrial world because of their preferences over the wired networks. Notwithstanding saving cabling costs, WSNs enlarge the domain of conditions attainable for checking. Consequently, adding inciting and detecting abilities to objects in the actual world and taking into account communication among them.

To additionally represent the structure of IWNs, a plant or industrial facility inside viewpoint is utilized as appeared in Figure 1 beneath. Accordingly, the communications framework can be isolated into four parts: brilliant elements, between IWNs, past IWNs, displayers, and workers. Inside IWNs, shrewd elements, for example, laborers, AGVs, machines, and standard sensors with wireless handsets could be viewed as wireless hubs that are associated with structure an IWN by wireless radios. Moreover past IWNs, the passage hubs and the door make a scaffold to different networks, for example, cell, and wired, and so forth More significant level data applications including data workers, the executives, regulators, and displayers might be founded on these particular networks Having the option to choose a reasonable wireless technology doesn't just need the information on the names of existing wireless technologies and their necessities yet additionally an away from of every wireless technology, its set of experiences, advancement, suggested applications, and so forth is required. As per (Lee, Su, and Shen 2007), The short-range wireless scene is as of now held by five protocols, Bluetooth, Wireless HART, ZigBee, ISA100.11a, and Wi-Fi that relate to the IEEE 802.15.1, 802.15.3, 802.15.4, and $802.11 \mathrm{a} / \mathrm{b} / \mathrm{g}$ norms, individually. The IEEE principles characterize the physical (PHY) and medium access control (MAC) layers for wireless communications over an activity scope of around 10-100 meters. Moreover, the principles Wireless HART and ISA100.11a were created by the HART communication establishment and the global society of robotization (ISA), individually. Each of the previously mentioned have various ascribes/highlights that decides their appropriateness of satisfying the prerequisites of various industrial frameworks as far as, dormancy, data rate, jitter, dependability, communication traffic conditions, situations/applications, network topologies, Range, bandwidth, and so on 


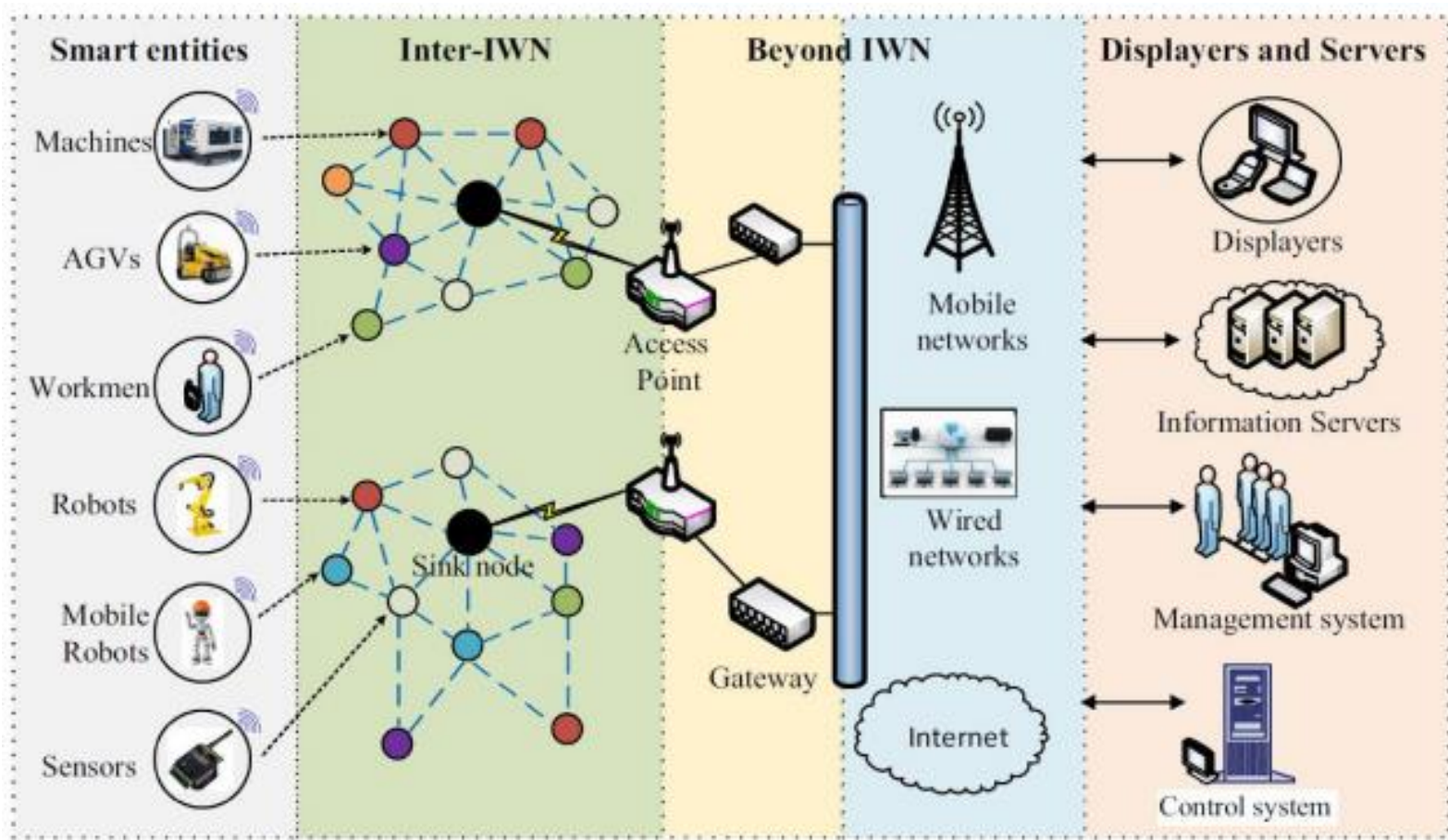

Figure 1. Industrial wireless network schematic diagram

\section{Bluetooth}

Bluetooth, which compares to the IEEE 802.15.1 depends on a wireless radio framework intended for short reach, modest communication devices reasonable for subbing links for printers, consoles, mice, and so forth The devices could likewise be utilized for communications between compact PCs, and go about as scaffolds between different networks, or fill in as hubs of specially appointed networks (characterize) that are considered multibounce wireless networks where a bunch of hubs collaborate to keep up the network availability (Flammini et al. 2009). This scope of uses is known as WPAN (Wireless Personal Area Network). Bluetooth is at present at adaptation 2.0. since walk 2004, the IEEE 802.15 working gathering has embraced the turn out accomplished for Bluetooth and made it an IEEE standard, specifically IEEE 802.15.1 (Ferro and Potorti 2005; Lee, Su, and Shen 2007; Burg, Chattopadhyay, and Lam 2018; Ahmadi et al. 2018). Two network topologies, the piconet and the scatternet are characterized in Bluetooth. A piconet, which follows a star geography as appeared in Figure 2.4 is a Wireless individual region network (WPAN) framed by a Bluetooth gadget filling in as an expert in the piconet and at least one Bluetooth devices filling in as slaves (Burg, Chattopadhyay, and Lam 2018; Georgakakis et al. 2010; Pothuganti and Chitneni 2014; Frotzscher et al. 2014). A recurrence bouncing channel dependent on the location of the expert characterizes each piconet.

Zigbee

ZigBee is a particular for a practical, low-rate, and low-power wireless networking technology kept up and distributed by a gathering of organizations known as the ZigBee Alliance (Lennvall, Svensson, and Hekland 2008; Ahmadi et al. 2018). It is viewed as a norm for wireless networks with low pinnacle throughput necessities and for short-range application zones, for example, home networks, distant checking, and control (Burg, Chattopadhyay, and Lam 2018; Gomez and Paradells 2010; Zhang and Shin 2011). Its convention stack is made out of four fundamental layers: the actual layer (PHY), the medium access control (MAC) layer, the network layer (NWK), and the application layer (APL). Moreover, ZigBee gives security usefulness across layers. In addition, the physical (PHY) and the medium access control (MAC) layers are characterized by the IEEE 802.15.4 norm, and the remainder of the stack is characterized by the ZigBee partnership. ZigBee is intended to oblige communication traffic types that are described by short unpredictable overflows with long resting periods. This trademark permits the leaf hubs of the network to work for a very long time or years on a solitary battery charge. A ZigBee network can likewise oblige an exceptionally enormous number of hubs of up to 65,000 hubs, that can be organized in either a star, tree, or lattice geography with various excess switches that broadens the network territory past the scope of a solitary highlight point connect (Burg, Chattopadhyay, and Lam 2018; Abinayaa and Jayan 2014; Ahmadi et al. 2018).

Wi-Fi

Wi-Fi represents Wireless Fidelity, which alludes to wireless technology that permits devices to impart over a wireless sign (Tan, Sooriyabandara, and Fan 2011; Ahmadi et al. 2018). Wireless neighborhood are ubiquitous in home, office, and in industrial conditions. It utilizes waves to permit rapid data moves over short distances 
permitting its clients to ride the web at broadband paces when associated with a passageway (AP) or in specially appointed mode. Also, the network depends on the IEEE 802.11; including 802.11a, 802.11b, 802.11g, and $802.11 \mathrm{n}$ working in either $2.4 \mathrm{GHz}$ or the $5 \mathrm{GHz}$ band reliant on which correction is utilized (Li et al. 2017). Various revisions $(\mathrm{a} / \mathrm{b} / \mathrm{g} / \mathrm{h})$ speak to the different strides in the standard development equipped towards improving throughput driven by the necessities of various use applications. Also, the IEEE 802.11 engineering comprises of a few segments that collaborate to give wireless Local Area Network (LAN) that supports station portability with bandwidth recurrence that advanced from initially $20 \mathrm{MHz}$ to $40 \mathrm{MHz}$ with the expansion of more complex tweak plans to help data rates between $11 \mathrm{Mb} / \mathrm{s}$ for IEEE $802.11 \mathrm{~b}$ up to $144 \mathrm{Mb} / \mathrm{s}$ for IEE $802.11 \mathrm{n}$ (Burg, Chattopadhyay, and Lam 2018; Tan, Sooriyabandara, and Fan 2011; Ahmadi et al. 2018).

\section{The benefits of WiFi}

Combined with minimal effort wireless sensors, WiFi can be utilized to gauge non-conventional cycle factors (not simply stream, level, pressing factor and temperature). WiFi can be utilized to successfully supplant wired Ethernet networking in regions where running wires is troublesome, costly or unthinkable. There are numerous spots in the industrial climate where that is the situation.

Wireless is being utilized right now for a wide assortment of utilizations. It makes re-designing sequential construction systems a lot less difficult and significant degrees more affordable if the re-setup doesn't likewise incorporate re-wiring each and every sensor to each and every regulator. There are wireless connector frameworks for each assortment of programmable regulator, regardless of whether PLC or PAC, or installed regulator.

$\mathrm{WiFi}$ is as of now in like manner use for programmed guided vehicles (AGVs). AGVs move stock around plants from area to area without human intercession, in view of their wireless communications. AGVs take crude materials to the creation zone, and take completed merchandise to stock. They are guided by 802.11-based control signals

The key to wireless in the plant is IEEE 802.11, the WiFi standard.

$802.11 \mathrm{n}$ incorporates numerous upgrades to the prior $\mathrm{a} / \mathrm{b} / \mathrm{g}$ renditions of the convention. These upgrades remember a speed up, reach and dependability.

Version $\mathrm{g}$ and $\mathrm{b}$ work at the $2.4 \mathrm{GHz}$ recurrence and a works at $5 \mathrm{GHz}$, the benefit of $\mathrm{n}$ is that it can work at both while likewise having the option to help synchronous $802.11 \mathrm{a}$ and $802.11 \mathrm{n}$ at $5 \mathrm{GHz}$ or $802.11 \mathrm{~b}$, $\mathrm{g}$ and $\mathrm{n}$ at $2.4 \mathrm{GHz}$. For use in Greenfield destinations the passage can be arranged not to be in reverse viable with different variants. To improve the reach, throughput and unwavering quality of the wireless networks $802.11 \mathrm{n}$ had made three center advancements: MIMO, parcel accumulation and channel holding, in this manner giving a fivefold expansion in execution over $802.11 \mathrm{a} / \mathrm{b} / \mathrm{g}$ networks.

MIMO frameworks are assembled utilizing different vector recieving wires at both the transmitter and the collector and it is this assortment that makes a MIMO framework. Since it can use both the variety and muxing of concurrent data streams it can conceivably expand framework limit by multiple times or more and has been embraced into IEEE 802.11n Depending on where MIMO signals are prepared a MIMO framework can be characterized into three kinds: recipient handling just, transmitter handling just, both transmitter and beneficiary preparing frameworks.

\section{Industrial Grade Wireless}

Because 802.11 is a COTS (business off the rack) standard doesn't imply that COTS items can be utilized in the industrial climate. Industrially solidified passages like the EKI-6311GN will make due in the industrial climate when presented to limits of temperature, dust, dampness, unsafe fumes and other regular circumstances.

\section{Mesh Technology}

A Mesh network geography, viewed as one of three assortments of specially appointed network for example one without a previous framework \{mobile (MANET), and wireless (WSN) are the other two \} necessitates that every hub should catch and disperse its own data, yet in addition fill in as a hand-off for different hubs for example It should team up in the engendering of data in the network.

A mesh network can be planned utilizing the steering procedure. Directing spreads the message along a way, by jumping from hub to hub until the objective is reached. To guarantee the accessibility of a way, a steering network should consider a nonstop association and reconfiguration around broken or impeded ways, utilizing self-mending calculations. Self-recuperating capacities empower directing based networks to work when one hub separates or an association turns sour. Therefore, the network is viewed as entirely dependable since there is frequently more than one way between a source and an objective in the network.

\section{Security and Risk in Wi-Fi}

Radio is dependent upon obstruction and sign quality issues that don't confront specialists of wired networking. Issues of reach, recieving wire plan and arrangement, impedance, meandering, and signal quality should be managed. In the workplace climate, wireless for the most part works essentially and without any problem. Conditions seldom change, so the network is entirely steady and not disappointment inclined. In the industrial climate, in any case, wireless communications are not exactly so straightforward. There is clamor (electrical 
commotion, EMF) which can meddle with radio stations. Welding, for instance, radiates commotion in similar groups as wireless networks use. PDAs and walkie-talkies produce obstruction in the WiFi groups, also.

The industrial climate frequently comprises of enormous bits of apparatus, metal structures, tanks and vessels that are very difficult to engender radio waves through. What's more, dispersing and bobbing radio signs off these structures causes a particular sort of impedance called "multipath" which is the explanation that FM radio some of the time sounds fluffy or has dropouts. This is called picket-fencing signals, in light of the fact that the sign travels every which way with the consistency of a picket fence. Multipath is caused when the sign takes at least two ways to arrive at the recipient, and relying upon the length of the ways, stage retraction is delivered and the sign goes to zero. One of the contemplations in choosing wireless devices is yield power. More force completes two things. To begin with, clearly, it releases the sign farther. In any case, higher force additionally permits the sign to slice through substantially more commotion, and in the industrial climate with heap wellsprings of RF and electrical work, that is a key component. The best and least complex approach to fix an obstruction issue is to either stop the wellspring of the impedance or move the radio to where the obstruction doesn't overwhelm or pulverize the sign. The accessibility of "spans" can be utilized to put an extra switch where it tends to be view from the primary switch to the following, in this manner eliminating the obstruction. In the industrial climate, the utilization of high sign strength $802.11 \mathrm{n}$ based devices and the utilization of 802.11a on the $5 \mathrm{GHz}$ band goes far to limit impedance.

\section{Conclusion}

Wireless networks are possibly unreliable, yet critical work has been done to improve the security of the 802.11 networks. WPA and WPA2 encryption are the current norm for security of a wireless transmission, and it is considered incredibly solid, when went with an exceptionally solid passphrase. WiFi based networks in the plant climate ought to cling to great security works on, including utilizing DMZs and firewalls, similarly as in the venture office climate. The utilization of port-based validation, in view of the $802.1 \mathrm{x}$ norm, and the utilization of RADIUS verification workers on top of WAP2 encryption make it amazingly hard to infiltrate an industrial wireless network.

\section{REFERENCES}

1. Rawat, P.; Deep Singh, K.; Chaouchi, H.; Bonnin, J.M. Wireless sensor networks: A survey on recent developments and potential synergies. J. Supercomput. 2014, 68, 1-48.

2. Jawad, H.M.; Nordin, R.; Gharghan, S.K.; Jawad, A.M.; Ismail, M. Energy-Efficient wireless sensor networks for precision agriculture: A review. Sensors 2017, 17, 1781.

3. Yu, X.; Wu, P.; Han, W.; Zhang, Z. A survey on wireless sensor network infrastructure for agriculture. Comput. Stand. Interfaces 2013, 35, 59-64.

4. Du, Y.-C.; Lee, Y.-Y.; Lu, Y.-Y.; Lin, C.-H.;Wu, M.-J.; Chen, C.-L.; Chen, T. Development of a telecare system based on ZigBee mesh network for monitoring blood pressure of patients with hemodialysis in health care centers. J. Med. Syst. 2011, 35, 877-883.

5. Medina, J.; Sánchez-Rodríguez, T.; Gómez-Galán, J.A.; Delgado, A.; Gómez, F.; Jiménez, R. A wireless sensor system for real-time monitoring and fault detection of motor arrays. Sensors 2017, 17, 677-684.

6. Alippi, C.; Camplani, R.; Galperti, C.; Roveri, M. A robust, adaptive, solar-powered WSN framework for aquatic environmental monitoring. IEEE Sens. J. 2011, 11, 45-55.

7. Kumar, A., Vengatesan, K., Rajesh, M., \& Singhal, A. (2019). Teaching literacy through animation \& multimediaInt. J. Innovative Technol. Exploring Eng, 8(5), 73-76.

8. Capella, J.V.; Bonastre, A.; Ors, R.; Peris, M. In line river monitoring of nitrate concentration by means of a wireless sensor network with energy harvesting. Sens. Actuators B 2013, 177, 419-427.

9. Anisi,M.H.; Abdullah, A.H. Efficient data reporting in intelligent transportation systems. Networks Spat. Econ. 2016, 16, 623-642.

10. Suryadevara, N.K.; Mukhopadhyay, S.C.; Tebje Kelly, S.D.; Singh Gill, S.P. WSN-based smart sensors and actuator for power management in intelligent buildings. IEEE/ASME Trans. Mecatronics 2015, 20, 564-571.

11. Rault, T.; Bouabdallah, A.; Challal, Y. Energy efficiency in wireless sensor networks: A top-down survey. Comput. Networks 2014, 67, 104-122.

12. Aparicio, J.; Echevarria, J.J.; Legarda, J.A. Software defined networking approach to improve the energy efficiency of mobile wireless sensor networks. IEEE Trans. Inter. Inf. Syst. 2017, 11, 2848-2869.

13. Ege, I.; Gökhan-Sensoy, M.; Kalender, O.; Nazlıbilek, S.; Citak, H. A new wireless asynchronous data communications module for industrial applications. Measurement 2013, 46, 2672-2681.

14. Yuan, Y.; Xu, Q.; Guan, X.; Liu, Z. Industrial high-speed wireless synchronous data acquisition system with real-time data compression. Measurement 2013, 46, 3482-3487.

15. Toscano, E.; Lo Bello, L. Multichannel superframe scheduling for IEEE 802.15.4 industrial wireless sensor networks. IEEE Trans. Ind. Inform. 2012, 8, 337-350. 
16. Kone, C.T.; Hafid, A.; Boushaba, M. Performance management of IEEE 802.15.4 wireless sensor network for precision agriculture. IEEE Sens. J. 2015, 15, 5734-5747.

17. Ambeth Kumar, V., Malathi, S., Venkatesan, R., Ramalakshmi, K., Vengatesan, K., Ding, W., \& Kumar, A. (2020). Exploration of an innovative geometric parameter based on performance enhancement for foot print recognitionJournal of Intelligent \& Fuzzy Systems(Preprint), 1-16 